\title{
1 Cryptic Dispersal Networks Connect Habitat Patches in ways not Captured
}

\section{2 by Distance}

4 Authors: Rachel M. Germain ${ }^{1,3}$, Natalie T. Jones ${ }^{2,3}$, Tess N. Grainger ${ }^{3}$

6 Affiliations:

$7{ }^{1}$ Biodiversity Research Centre, University of British Columbia, Vancouver, British Columbia,

8 Canada

$9{ }^{2}$ Department of Ecology, Behavior and Evolution, University of California San Diego, San

10 Diego, CA, USA

$11{ }^{3}$ Department of Ecology and Evolutionary Biology, University of Toronto, Toronto, ON, Canada

13 *Corresponding author: rgermain@zoology.ubc.ca

14 Review process: The authors wish to be identified to reviewers.

16 Keywords: connectivity, dispersal mode, fragmentation, invasion biology, McLaughlin Natural

17 Reserve, serpentine grassland

18 Manuscript type: Article

20 Manuscript elements: Abstract [179], Introduction [1151], Methods [1583], Results \&

21 Discussion [1444], Conclusion [139], Acknowledgements [43], Figures [3], Tables [1],

22 Appendices A and B 


\section{Abstract}

25 Species interact with the physical world in complex ways, and life history strategies might cause

26 species to differ in how they experience connectedness of the same landscape. As a consequence,

27 dispersal limitation might be present but not captured by distance-based measures of

28 connectivity. To test these ideas, we surveyed plant communities that associate with serpentine

29 soils but differ in dispersal mode (gravity, animal, or wind), and used satellite imagery to

30 quantify forms of landscape connectivity associated with each dispersal mode. Our data yielded

31 two key insights: First, dispersal limitation appeared to be absent using a conventional distance-

32 based measure of connectivity, but emerged after considering forms of landscape connectivity

33 relevant to each dispersal mode. Second, the landscape variables that emerged as important to

34 each dispersal mode were generally consistent with our predictions based on putative dispersal

35 vectors, and included interactive effects that allude to the altered efficacy of animal dispersal in

36 invaded landscapes. Our results have broad implications for understanding how ecological

37 communities reorganize as landscapes are fragmented, patches are lost, and the function of

38 dispersal life histories is altered. 


\section{Introduction}

48 Ecologists have long-sought to quantify the importance of dispersal limitation in ecological

49 communities (Borcard et al. 2004; Gilbert and Lechowicz 2004; Cottenie 2005) for two main

50 reasons. First, the presence of dispersal limitation can cause local species richness to fall short of

51 what a given environment can support (Germain et al. 2017), and second, the failure of

52 propagules to reach suitable sites generates spatial turnover in species richness and composition

53 that contribute to regional biodiversity (Hurtt and Pacala 1995; Mouquet and Loreau 2003).

54 Towards this goal, numerous statistical tools have been developed to isolate the relative

55 explanatory power of dispersal vs. environment from field data (e.g., (Peres-Neto et al. 2006;

56 Prugh 2009)), typically testing for and interpreting an effect of distance among local

57 communities on species occupancy, richness, or composition as evidence of dispersal limitation

58 (Hanski 1994a; Cottenie 2005; Prugh et al. 2008). If spatial distance among sites is assumed to

59 be the best proxy of restricted dispersal, then the absence of significant distance effects on

60 diversity patterns is interpreted as evidence that dispersal is not limiting at the spatial scales

61 examined (e.g., (Freestone and Inouye 2006)) - that is, that species have access to all habitat

62 patches, and that variation in species occupancy and richness patterns reflect variation in local

63 environmental conditions.

64 An alternative but rarely considered explanation for non-significant distance effects is

65 that isolation by distance is not the spatial variable most relevant to dispersal - habitat patches

66 might be close in space but poorly connected by dispersal due to other landscape features, such

67 as physical barriers. Though this idea has been explored in aquatic ecosystems for which there is

68 obvious network structure (e.g., riverine networks (Beisner et al. 2006; Brown and Swan 2010))

69 or directionality to dispersal (e.g., water currents (White et al. 2010)), it has not been explored in 
70 terrestrial systems for which dispersal barriers may be cryptic and thus difficult to identify and

71 measure. Although isolation by distance is likely the most important factor limiting dispersal in

72 many ecosystems (e.g., oceanic islands), exploring alternative dispersal pathways can reveal

73 hidden constraints to how species move across and interact with their landscapes, and might

74 explain why distance effects are generally weak in terrestrial ecosystems; a recent synthesis of

751,015 animal surveys found that spatial isolation was a poor predictor of patch occupancy for

76 most species (Prugh et al. 2008).

If dispersal is constrained in a greater range of ways other than by distances among

78 habitat patches, then, intriguingly, species might differ in how they experience the spatial

79 connectedness of the same physical landscape based on dispersal life histories (Beisner et al.

80 2006). In plants, for example, species possess a range of adaptations to disperse, called dispersal

81 syndromes or "modes", such as dispersal by gravity, animals, or wind. Previous research of

82 understory herbs in aspen stands clearly demonstrates that dispersal mode dictates how

83 constrained plant species distributions are by the size and spatial isolation of habitat patches,

84 even without accounting for additional sources of trait variation (e.g., seed size) among species

85 within dispersal modes (Jones et al. 2015). In that study, however, the effect of dispersal mode

86 on species distributions was not consistent with a simple difference in dispersal ability among

87 modes (i.e., dispersal ability: gravity < wind < animal (Jones et al. 2015)), as hypothesized if

88 distances among habitat patches was the only cause of spatial isolation. We contend that linking

89 species distributions to the spatial distribution of dispersal vector movement might be the

90 missing piece needed to understand the mechanisms that underlie the spatial distribution and

91 composition of biodiversity, for plants and potentially other terrestrial organisms. Identifying

92 spatial constraints on species distributions is key to understanding the processes that underlie 
93 fundamental patterns in ecology, such as species-area relationships (Shen et al. 2009), as well as

94 to forecast how ecological communities might reorganize as the spatial and environmental

95 structure of landscapes is altered by humans (Gonzalez et al. 2011; Frishkoff et al. 2016).

96 We explored alternative forms of landscape connectivity to understand the distribution of

97 biodiversity in a natural patch-network of plants that associate with serpentine soils. Serpentine

98 soils form via the emergence and erosion of the Earth's mantle into discrete patches embedded

99 within a matrix of non-serpentine soil. Serpentine soils are hypothesized to act as "islands" of

100 refuge for native plant species to escape the "sea" of European grasses that now dominate

101 Californian landscapes (Harrison and Rajakaruna 2011; Gilbert and Levine 2013). The annual

102 plant communities that associate with serpentine soils are an emerging model system to

103 understand the mechanisms that underlie the spatial scaling of biodiversity (Anacker and

104 Harrison 2012; Germain et al. 2017), the interaction between local and regional processes

105 (Harrison 1999; Harrison et al. 2006), and the community impacts of species invasions (Gilbert

106 and Levine 2013; Case et al. 2016). Recent experimental work has demonstrated that dispersal

107 limits plant diversity at our study site (fig. 1), yet we find no evidence of spatial distance as a

108 proxy for dispersal limitation through our observational data (table A2); this contradiction

109 motivates our examination of other landscape features relevant to dispersal. Specifically, the

110 absence of tall vegetation in serpentine grasslands allowed landscape features, such as

111 hydrological networks and animal paths, to be captured via satellite imagery (fig. 2).

112 We surveyed plant communities on serpentine patches and in the non-serpentine matrix,

113 categorized species by dispersal mode, and estimated habitat patch characteristics relevant to

114 different modes of dispersal. We used these data to answer two questions: (1) Are there

115 landscape features that characterize habitat patch connectivity better than distance among habitat 
116 patches, and (2) does dispersal mode influence how species respond to these features? If species

117 experience different landscape constraints, then we predict that the richness of species belonging

118 to different dispersal modes will be highest in habitat patches highly connected by their

119 respective dispersal vectors. Specifically, we predict that hydrological networks, animal paths,

120 and distance would explain the richness of species dispersed by gravity, animals, and wind,

121 respectively. As a case study, we also explore the spatial distribution of patch occupancy patterns

122 of Plantago erecta (California plantain), a small-statured annual with seed morphologies

123 consistent with a mixed dispersal strategy (i.e., dispersal via water and animals (Germain et al.

124 2017)). If our models are correct, then we predict that $P$. erecta's distributions would be

125 explained by forms of habitat connectivity shown to be important to both dispersal modes.

126 Our analyses of species richness fall into a general class of 'incidence function' models

127 (Prugh 2009), the basis of which was first developed by Levins (Levins 1969) and later adapted

128 by Hanski (Hanski 1994a, 1994b) to test species' extinction and colonization as a function of

129 patch size and isolation by distance, respectively. These models have achieved broad success at

130 understanding the population and metapopulation persistence of a diversity of organisms in

131 fragmented landscapes (e.g., butterflies [28], pikas (Moilanen et al. 1998)), with applications to

132 landscape management and conservation planning (Wahlberg et al. 1996).

Materials and Methods

Study System

136 Our study took place at the 2800-ha McLaughlin Natural Reserve (http://nrs.ucdavis.edu/mcl/) in

137 Northern California, at the boundary of Lake, Yolo, and Napa counties (3851'47.01"N,

$\left.138 \quad 122^{\circ} 21^{\prime} 48.87^{\prime \prime} \mathrm{W}\right)$. The landscape is characterized by patches of serpentine soil interspersed 
among a matrix of non-serpentine soil. Serpentine (ultramafic) soils are derived from the Earth's

140 mantle in regions where it becomes exposed, such as along the San Andreas Fault, and are

141 identified by $\mathrm{Ca} / \mathrm{Mg}$ ratios < 1 (Anacker 2014). Calcium is essential to plant growth, and is

142 captured less efficiently in the presence of magnesium. Low $\mathrm{Ca} / \mathrm{Mg}$ ratios, coupled with low soil

143 fertility, high heavy metal content, and poor soil moisture retention, present a harsh growing

144 environment for plants. Yet, serpentine soils support a rich diversity of native and endemic plant

145 species (Anacker 2014), and are hypothesized to act as spatial refugia for native species to

146 escape the competitive effects of the exotic European plants that now dominate the non-

147 serpentine matrix (Gilbert and Levine 2013).

Field Survey and Data Collection

150 Plant surveys and all fieldwork were conducted in early May 2017, at approximately peak

151 flowering. We haphazardly selected 28 serpentine habitat patches out of all 42 patches in a 18 ha

152 region of the reserve, ranging from 31 to $4533 \mathrm{~m}^{2}$ in size and 0.75 to $356 \mathrm{~m}$ away from their

153 nearest neighbor patch (fig. 2). At each patch, we surveyed a transect of five $0.75 \times 0.75 \mathrm{~m}^{2}$

154 plots: one plot in the patch centre, one plot halfway between each edge and the patch centre, one

155 plot $1 \mathrm{~m}$ into the non-serpentine matrix, and one plot $5 \mathrm{~m}$ into the matrix (fig. A1). In other

156 words, the distances among plots within patches were scaled by patch size, whereas the two

157 matrix plots were fixed distances from the patch edge. We recorded the presences of all species

158 in each plot, and made note of species that covered more than $25 \%$ of a plot by area (usually one

159 to three species). In total, 77 plant species were present in our surveys, 72 of which could be

160 identified; the five unidentified species occurred once each, had no distinguishing features to

161 assess dispersal mode with certainty (i.e., only a single basal leaf) and were discarded from all 
162 analyses that required information on dispersal mode. Sampling the same total area for all habitat

163 patches regardless of patch size is a standard sampling method to prevent confounding patch size

164 with sampling intensity (Cook et al. 2002).

165 Species' dispersal modes (dispersal via wind, gravity, or animals) were categorized based

166 on previous research (Spasojevic et al. 2014) and updated here based on seed/diaspore

167 morphology and if more detailed information on dispersal modes was available (table A1).

168 Wind-dispersed species were identified by the presence of a pappus or seed wings, whereas

169 animal-dispersed species had morphologies for attachment to passing animals, such as burrs,

170 awns, or hairs. Species categorized as gravity dispersed had seeds that lacked any apparent

171 mechanism for dispersing by wind or animals, and tended to have smooth, spherical diaspores

172 conducive to downslope dispersal via rain and gravity. We include ant-dispersed species as

173 gravity dispersed given that ants disperse seeds at very small spatial scales and are unlikely to

174 contribute to regional occupancy patterns (Thomson et al. 2011), as well as species with reduced

175 pappi that were biomechanically unlikely to confer wind dispersal (e.g., Lasthenia californica).

176 One species, Plantago erecta (California plantain), was previously categorized as being

177 dispersed by water (Spasojevic et al. 2014). However, P. erecta seeds produce a sticky mucilage

178 that might also allow dispersal by animals (observation noted in (Germain et al. 2017)). As such,

179 we categorize this species as being animal-dispersed but also explore the occupancy patterns of

180 this species in depth as a case study of a species with two potential dispersal modes.

181 Species were additionally categorized as patch- or matrix-associated (table A1) to

182 identify and account for species that were unlikely constrained to serpentine habitat patches

183 (Cook et al. 2002; Jones et al. 2015). 'Matrix-associated' species included both matrix specialists

184 and generalists that show no affinity for habitat type. Species were considered matrix-associated 
185 if they were equally or more common in matrix plots than in the patch plots. Twelve species met

186 these criteria, including Avena fatua, Bromus hordeaceus, and Lotus wrangelianus. Species

187 richness (fig. A2A) and composition (fig. A2B) differed among serpentine habitat patches and

188 the non-serpentine matrix (both $P<0.001$ ), confirming that serpentine plant communities are

189 distinct and thus constrained to the serpentine habitat-patch network.

190 We estimated habitat patch characteristics in the field and using ArcGIS on GoogleEarth

191 images. In ArcGIS, we delineated all serpentine patches within our study region, including the

19228 surveyed patches and 14 unsurveyed patches; these delineations allowed us to calculate patch

193 size and patch connectivity. Patch connectivity was estimated using edge-to-edge distances

194 between patch $i$ and all other $j$ patches (including the unsampled patches), weighted by a

195 negative exponential dispersal kernel using eq. 1 (Hanski 1994a, 1994b; Jones et al. 2015):

197 connectivity $_{i}=\sum_{j \neq i}^{n} A \cdot e^{-d_{i j} / \alpha}$

199 where $A$ is the area of patch $j, d$ is the Euclidean distance in meters between patch $i$ and $j$, and $\alpha$

200 is the mean dispersal distance, set to $5 \mathrm{~m}$ for all species. In other words, patch $i$ is most

201 connected when it is in close proximity to many large patches. Our connectivity measure falls

202 into a general class of measures called incidence function models, which have been shown to

203 perform equally well or better than alternative measures (i.e., nearest-neighbour or buffer

204 measures (Prugh 2009).

205 We estimated two alternative measures of connectivity that we hypothesized could be

206 more appropriate for plant species that are dispersed by animals or gravity. For species that are

207 dispersed by animals, we traced deer trails that were observable via GoogleEarth (fig. 2) using 
ArcGIS, and used the number of trails that intersected habitat patches to estimate patch connectivity via animals. Deer exhibit path fidelity, following the path of least resistance, which

210 is especially true in topographically challenging landscapes such as our study area, and create

211 "highways" for a diversity of other animals to traverse landscapes, including rabbits and turkeys

212 (Sindorf 2009). For plant species that are dispersed by gravity/water, we estimated hydrological

213 connectivity by tracing the network of surface streams, and for each habitat patch, summed the

214 area of all $j$ habitat patches upslope from and connected via surface streams to each patch $i$.

215 Elevation of habitat patches ranged 450-550 $\mathrm{m}$ a.s.1., small enough for elevational clines in

216 climate to be unimportant. Although in some systems, habitat patches at the base of an

217 elevational cline have increased resource inputs and thus higher productivity, productivity was

218 not correlated with elevation (slope $<0.01, P=0.465)$ or hydrological connectivity (slope = -

$2190.03, P=0.458)$ in our dataset. We estimated productivity as a composite measure [(1 -

220 proportion of bare ground) $\mathrm{x}$ vegetation height] to non-destructively estimate the volume of plant

221 material in each plot.

224 To test if species composition in serpentine habitat patches was distinct from the surrounding

225 non-serpentine matrix, we used linear mixed effects models to test differences in species

226 composition among plots in serpentine habitat patches ("patch plots"), $1 \mathrm{~m}$ into the habitat

227 matrix ("edge plots"), and $5 \mathrm{~m}$ into the habitat matrix ("matrix plots"). To do so, we first

228 performed a principal coordinates analysis using Jaccard's distances on the plot-level

229 presence/absence data. The first and second axis scores were used as response variables in

230 separate analyses with fixed effects of habitat type (i.e., patch, edge, matrix) and 'patch id' 
231 included as a random effect to account for the non-independence of the five plots transecting

232 each habitat patch. The 'glht' function in R package 'multcomp' was used to to perform a

233 Tukey's tests of compositional differences among all pairwise treatment combinations (patch vs.

234 edge, patch vs. matrix, edge vs. matrix).

235 To examine the relationship between species richness and patch connectivity, we

236 performed a generalized linear mixed effects model with species richness as the response, fixed

237 effects of dispersal mode, connectivity by distance, connectivity by animals, connectivity by

238 hydrology, patch size, and all interactions, and random effect of 'patch id' to account for non-

239 independence of replicate plots within a single patch. Because there were significant higher-

240 order interactions between dispersal mode and all connectivity measures (table A2), we

241 performed separate analyses of biogeographic predictors for each dispersal mode. This type of

242 analysis is highly prone to type I errors (poisson-distributed data with interacting continuous

243 predictors), so we took several steps to identify reduced models that best fit the data. First, we

244 performed backwards selection on each full model using the 'step' function in the 'stats'

245 package; 'step' sequentially drops higher order interactions until the reduced model that yields

246 the greatest model fit (lowest AIC score) is attained. However, 'step' can arrive at a local

247 minimum in AIC score that does not reflect the global minimum, which was likely for our data

248 given the presence of significant but biologically implausible four-way interactions. For this

249 reason, we applied 'drop1' to the 'step'-reduced model to identify variables that did not

250 significantly improve model fit even if their inclusion led to a marginal decrease in AIC scores.

251 We cycled between 'drop1' and 'step' until a model was obtained for which all variables

252 significantly improved model fit and led to the lowest AIC scores out of all possible reduced 
models. We then used the function 'Anova' in the R package 'car' to test whether the reduced

254 model was a significantly better fit to the data than an intercept-only model.

255

256

257

258

259

260

261

262

263

264

265

266

267

268

269

270

271

272

273

274

275

\section{Results and Discussion}

Despite the emphasis that contemporary ecology places on dispersal as central to the dynamics and distribution of species in ecological communities (Hanski 1994a; Leibold et al. 2004), current empirical assessments of its role may not encompass the diversity of ways in which organisms experience landscape connectivity. In a patchy terrestrial plant community, we found no evidence of dispersal limitation using a distance-based estimate of patch connectivity, even after discounting the presences of species associated with the habitat matrix (i.e., a nonsignificant effect of connectivity by distance; table A2), despite experimental evidence of its pervasiveness (fig. 1 with data from (Germain et al. 2017)). However, when separated species richness by dispersal mode (gravity, wind, animal), the spatial distributions of species richness generally corresponded to spatial patterns of dispersal vectors which connect habitat patches. We discuss these general findings, as well as several unexpected contingencies that provide a richer understanding of interacting dispersal vectors in serpentine grasslands and their altered efficacy in invaded landscapes.

Consistent with our prediction that the richness of gravity-dispersed species would be highest in patches highly connected by hydrology, hydrology was the only form of connectivity retained as a predictor after model selection for this group. However, the effect of hydrological connectivity was not simply a main effect, but rather, an interactive effect with patch size (i.e., significant hydrological connectivity $\times$ patch size effect $\left.\left[X^{2}=6.37, P=0.012\right]\right)$, such that species richness increased with each predictor only at low values of the other (i.e., fig. 3A, steep slopes connecting points 1 to 2 and points 1 to 4 , but shallow slopes connecting points 3 to 4 and points 
2772 to 3 ). Although we did not predict this interaction a priori, it suggests that large,

278 hydrologically-connected patches are locally saturated (i.e., response surface decelerates from

279 points 1 to 3 , note log-scale of axes) and that these two predictors act as compensatory pathways

280 towards reaching saturation. Our findings are consistent with recent experimental work showing

281 that dispersal only increases species richness in small habitat patches (Schuler et al. 2017), given

282 that populations in small patches are more prone to stochastic extinctions (Gilbert and Levine

283 2017) which can be overcome via dispersal.

The model that best fit the richness data of animal-dispersed species was one that, as

285 predicted, included the appropriate vector of dispersal: connectivity by animals. However, as

286 with gravity-dispersed species, the best-fit model also included an interaction, in this case

287 between connectivity by animals and connectivity by distance $\left(X^{2}=7.06, P=0.007\right)$, generating

288 a complex response surface (fig. 3B). More species were found in serpentine patches intersected

289 by many animal paths, but only when patches were in close proximity to one another (slope

290 connecting points 3 to 4 in fig. $3 B$ ) - when patches were isolated, however, animals had a

291 strongly negative effect (slope connecting points 1 to 2 ). What is driving the negative effect of

292 animals in isolated patches? The answer is not likely herbivory, given that the animal-dispersed

293 species in our dataset are generally tolerant of or well-defended against herbivory (e.g., grasses,

294 star thistle (table A1)) and given that connectivity by animals did not predict the richness of

295 wind-dispersed species, a highly palatable group (e.g., wild lettuce, dandelion (table A1)).

296 Rather, we contend that the answer has more to do with the efficacy of animals as dispersal

297 vectors in invaded landscapes. Seeds removed by animals in isolated habitat patches have a low

298 probability of (i) being deposited in other habitat patches, compared to the inhospitable matrix,

299 and of (ii) being rescued from extinction via dispersal from other patches; in other words, seeds 
are removed but not replaced. Additionally, many of the most noxious invaders in serpentine grasslands are animal dispersed (e.g., common wild oat [Avena fatua], barbed goatgrass

302 [Aegilops triuncialis]), adding the potential for invader impacts via animal-mediated dispersal

303 from the non-serpentine matrix, even if those invaders exist only as sink populations (Schreiber

304 and Kelton 2005). We argue that the negative effect of dispersal via animals is likely a

305 contemporary phenomenon, given that, prior to invasion by European grasses, a greater

306 proportion of the landscape was suitable to species that are now restricted to occur only on

307 serpentine patches (Gram et al. 2004; Gilbert and Levine 2013). Further support for this

308 hypothesis comes from our finding that species richness increases with connectivity by distance

309 only in patches highly connected by animals (slope connecting points 2 and 3), reinforcing

310 animals as dispersal vectors, connecting patches that would otherwise be unconnected reduce

311 close proximity.

Although we predicted that the richness of wind-dispersed species would increase with

313 connectivity by distance, given that wind moves in all directions in topographically complex

314 landscapes (McNider and Pielke 1984), such as our study site, we instead found that an intercept-

315 only model best fit the data. This finding has three possible explanations, the first being that

316 wind-dispersed species are simply not dispersal limited at the spatial scale of our surveys, and

317 the second being the possibility that we have not adequately captured spatial variation in the

318 movement of seeds by wind. Although we cannot weigh these two alternate explanations against

319 each other, what we can say is that there is a high degree of variation in species richness and

320 composition among patches for this dispersal group, including some patches that lack species

321 from this group altogether. High spatial turnover ( $\beta$ diversity) without evidence of dispersal

322 limitation implicates the role of local processes (Germain et al. 2013), such as environment, 
323 herbivory, competition, or stochasticity. However, a more detailed examination of dispersal

324 kernels and constraints for this group are needed.

325 The third explanation is that trait differences among wind-dispersed species, for example,

326 short vs. tall species (Thomson et al. 2011), caused additional variation in how species

327 experience landscape connectivity. Testing this possibility would require separate analyses of

328 species occupancy patterns for multiple species; our data is not amenable to such an analysis,

329 because only two wind-dispersed species occupied enough patches to reasonably fit an incidence

330 function model (MacKenzie et al. 2005). Coarsely, though, the most common wind-dispersed

331 species (Microseris douglasii), observed in 22 of the 28 sampled patches, was average in terms

332 of plant height and the ratio of seed size to dispersal structure, though did have the largest seeds

333 (fig. A4). Large-seeded wind-dispersed plant species disperse farther on average (Thomson et al.

334 2011), thus seed size differences may contribute to regional occupancy patterns for this group.

As predicted, the distribution of $P$. erecta, a common small-statured annual with seed

337 (Germain et al. 2017)), was explained by patch characteristics consistent with both dispersal

338 modes. Specifically, occupancy patterns of this species were influenced by a three-way

339 interaction between connectivity by distance, hydrology, and animals $\left(X^{2}=5.70, P=0.017\right)$, as

340 well as positive main effects of hydrology and animals (both $P \leq 0.001$; table 1 ). When patches

341 were well-connected by hydrology, the response surface of the probability that P. erecta was

342 present in patches resembled that of richness of animal-dispersed species (fig. A5B vs. fig. 3B).

343 However, when patches were poorly connected by hydrology, occurrence probabilities generally

344 increased with connectivity by animals (fig. A5A). This in-depth examination of single-species

345 occupancy patterns demonstrates consilience among approaches, where connectivity measures 
346 identified as important to different dispersal modes in the community level data also emerge as

347 important predictors of a species with a mixed dispersal strategy.

348 Habitat fragmentation is the primary driver of biodiversity loss worldwide (Crooks et al.

349 2011). In serpentine plant communities and many other ecosystems, fragmentation has occurred

350 via the widespread invasion of non-native species, with native species now relegated to small

351 isolated "refuge" habitat patches. Though species in refuge patches may be safe from direct

352 competition with invaders, diversity is still challenged with the indirect effects of reduced

353 colonization (Gilbert and Levine 2013). The extreme harshness of the competitive effect in the

354 non-serpentine matrix is clear if we consider that (i) plots in the non-serpentine matrix were 7.1

355 times more productive than serpentine plots yet contained 2.2 fewer species on average (fig.

$356 \mathrm{~A} 2 \mathrm{~A}$ ), and that (ii) there was no difference in species composition among plots $1 \mathrm{~m}$ vs. $5 \mathrm{~m}$ into

357 the matrix (grey vs. white points in fig. A2B) even though $1 \mathrm{~m}$ is within the dispersal capacities

358 of most species. In order to prevent the non-random loss of some species over others (e.g., plants

359 dispersed by animals), landscape management plans may need to consider alternate multiple

360 forms of habitat connectivity. Californian landscapes were invaded $\sim 200$ years ago, meaning that

361 current communities may already reflect the compositional reorganization of some groups over

362 others, a hypothesis that can be tested experimentally.

\section{Conclusion}

365 Characterizing habitat connectivity is fundamental to understanding how dispersal contributes to

366 biodiversity patterns (Leibold et al. 2004), as well as to landscape planning for conservation

367 (Crooks and Sanjayan 2006). In a serpentine grassland, we uncover cryptic dispersal networks by

368 linking species' dispersal life histories to dispersal vector movement. Our results suggest that 
369 ecologists should more carefully consider whether the absence of significant distance effects

370 truly represents an absence of dispersal limitation $v s$. a failure to capture landscape variables that

371 are most limiting to dispersal. Additionally, our finding that animal dispersal reduced diversity in

372 isolated habitat patches points towards the altered functioning of ecological networks in invaded

373 landscapes. Real landscapes include complex spatial flows of energy and matter, which as we

374 demonstrate, sets up ecological opportunity for organisms to differ in how they interact with and

375 experience the same landscape.

\section{Acknowledgements}

378 We thank Diane Srivastava and the Srivastava lab for comments on the manuscript, as well as

379 Roi Holzman for references on connectivity in the marine literature. Research funding was

380 provided to R.M.G. by the Biodiversity Research Centre at UBC and the Killam Trust.

\section{Author's Contributions}

383 RMG conceived of the study, RMG, NTJ, TNG designed the sampling design and performed

384 fieldwork, NTJ did the GIS, RMG and NTJ analyzed the data, RMG, NTJ, TNG wrote the paper.

\section{Literature Cited}

387 Anacker, B. L. 2014. The nature of serpentine endemism. American Journal of Botany 101:219388224.

389 Anacker, B. L., and S. P. Harrison. 2012. Historical and ecological controls on phylogenetic

390 diversity in Californian plant communities. The American Naturalist 180:257-269.

391 Beisner, B. E., P. R. Peres-Neto, E. S. Lindström, A. Barnett, and M. L. Longhi. 2006. The role 
392 of environmental and spatial processes in structuring lake communities from bacteria to fish.

393 Ecology 87:2985-2991.

394 Borcard, D., P. Legendre, C. Avois-Jacquet, and H. Tuomisto. 2004. Dissecting the spatial

395 structure of ecological data at multiple scales. Ecology 85:1826-1832.

396 Brown, B. L., and C. M. Swan. 2010. Dendritic network structure constrains metacommunity

397 properties in riverine ecosystems. Journal of Animal Ecology 79:571-580.

398 Case, E. J., S. Harrison, and H. V. Cornell. 2016. After an invasion: understanding variation in

399 grassland community recovery following removal of a high-impact invader. Biological Invasions

$400 \quad 18: 371-380$.

401 Cook, W. M., K. T. Lane, B. L. Foster, and R. D. Holt. 2002. Island theory, matrix effects and

402 species richness patterns in habitat fragments. Ecology Letters 5:619-623.

403 Cottenie, K. 2005. Integrating environmental and spatial processes in ecological community

404 dynamics. Ecology Letters 8:1175-1182.

405 Crooks, K. R., C. L. Burdett, D. M. Theobald, C. Rondinini, and L. Boitani. 2011. Global

406 patterns of fragmentation and connectivity of mammalian carnivore habitat. Philosophical

407 Transactions of the Royal Society of London B: Biological Sciences 366:2642-2651.

408 Crooks, K. R., and M. Sanjayan. 2006. Connectivity Conservation. Cambridge University Press.

409 Freestone, A. L., and B. D. Inouye. 2006. Dispersal limitation and environmental heterogeneity

410 shape scale-dependent diversity patterns in plant communities. Ecology 87:2425-2432.

411 Frishkoff, L. O., D. S. Karp, J. R. Flanders, J. Zook, E. A. Hadly, G. C. Daily, and L. K.

412 M'Gonigle. 2016. Climate change and habitat conversion favour the same species. Ecology

413 Letters 19:1081-1090.

414 Germain, R. M., L. Johnson, S. Schneider, K. Cottenie, E. A. Gillis, and A. S. MacDougall. 
415 2013. Spatial variability in plant predation determines the strength of stochastic community

416 assembly. The American Naturalist 182:169-179.

417 Germain, R. M., S. Y. Strauss, and B. Gilbert. 2017. Experimental dispersal reveals

418 characteristic scales of biodiversity in a natural landscape. Proceedings of the National Academy

419 of Sciences of the United States of America 114:4447-4452.

420 Gilbert, B., and M. J. Lechowicz. 2004. Neutrality, niches, and dispersal in a temperate forest

421 understory. Proceedings of the National Academy of Sciences of the United States of America

$422 \quad 101: 7651-7656$.

423 Gilbert, B., and J. M. Levine. 2013. Plant invasions and extinction debts. Proceedings of the

424 National Academy of Sciences of the United States of America 110:1744-1749.

425 2017. Ecological drift and the distribution of species diversity. Proceedings of the Royal

426 Society of London B: Biological Sciences 284:20170507.

427 Gonzalez, A., B. Rayfield, and Z. Lindo. 2011. The disentangled bank: how loss of habitat

428 fragments and disassembles ecological networks. American Journal of Botany 98:503-516.

429 Gram, W. K., E. T. Borer, K. L. Cottingham, E. W. Seabloom, V. L. Boucher, L. Goldwasser, F.

430 Micheli, et al. 2004. Distribution of plants in a California serpentine grassland: are rocky

431 hummocks spatial refuges for native species? Plant Ecology 172:159-171.

432 Hanski, I. 1994a. Patch-occupancy dynamics in fragmented landscapes. Trends in Ecology \&

433 Evolution 9:131-135.

434 1994b. A practical model of metapopulation dynamics. Journal of Animal Ecology

$435 \quad 63: 151-162$.

436 Harrison, S. 1999. Local and regional diversity in a patchy landscape: native, alien, and endemic

437 herbs on serpentine. Ecology 80:70-80. 
438 Harrison, S., K. F. Davies, H. D. Safford, and J. H. Viers. 2006. Beta diversity and the scale-

439 dependence of the productivity-diversity relationship: a test in the Californian serpentine flora.

$440 \quad$ Journal of Ecology 94:110-117.

441 Harrison, S., and R. Rajakaruna, eds. 2011. Serpentine: The Evolution and Ecology of a Model

442 System. University of California Press, Berkeley.

443 Hurtt, G. C., and S. W. Pacala. 1995. The consequences of recruitment limitation: reconciling

444 chance, history and competitive differences between plants. Journal of Theoretical Biology

$445 \quad 176: 1-12$.

446 Jones, N. T., R. M. Germain, T. N. Grainger, A. M. Hall, L. Baldwin, and B. Gilbert. 2015.

447 Dispersal mode mediates the effect of patch size and patch connectivity on metacommunity

448 diversity. Journal of Ecology 103:935-944.

449 Leibold, M. A., M. Holyoak, N. Mouquet, P. Amarasekare, J. M. Chase, M. F. Hoopes, R. D.

450 Holt, et al. 2004. The metacommunity concept: a framework for multi-scale community ecology.

451 Ecology Letters 7:601-613.

452 Levins, R. 1969. Some demographic and genetic consequences of environmental heterogeneity

453 for biological control. Bulletin of the Entomological Society of America 15:237-240.

454 MacKenzie, D. I., J. D. Nichols, N. Sutton, K. Kawanishi, and L. L. Bailey. 2005. Improving

455 inferences in population studies of rare species that are detected imperfectly. Ecology 86:1101-

4561113.

457 McNider, R. T., and R. A. Pielke. 1984. Numerical simulation of slope and mountain flows.

458 Journal of Climate and Applied Meteorology 23:1441-1453.

459 Moilanen, A., A. T. Smith, and I. Hanski. 1998. Long-term dynamics in a metapopulation of the 460 American pika. The American Naturalist 152:530-542. 
461 Mouquet, N., and M. Loreau. 2003. Community patterns in source-sink metacommunities. The

462 American Naturalist 162:544-557.

463 Peres-Neto, P. R., P. Legendre, S. Dray, and D. Borcard. 2006. Variation partitioning of species

464 data matrices: estimation and comparison of fractions. Ecology 87:2614-2625.

465 Prugh, L. R. 2009. An evaluation of patch connectivity measures. Ecological Applications

466 19:1300-1310.

467 Prugh, L. R., K. E. Hodges, A. R. E. Sinclair, and J. S. Brashares. 2008. Effect of habitat area

468 and isolation on fragmented animal populations. Proceedings of the National Academy of

469 Sciences of the United States of America 105:20770-20775.

470 Schreiber, S. J., and M. Kelton. 2005. Sink habitats can alter ecological outcomes for competing

471 species. Journal of Animal Ecology 74:995-1004.

472 Schuler, M. S., J. M. Chase, and T. M. Knight. 2017. Habitat patch size alters the importance of

473 dispersal for species diversity in an experimental freshwater community. Ecology and Evolution

$474 \quad 7: 5774-5783$.

475 Shen, G., M. Yu, X.-S. Hu, X. Mi, H. Ren, I.-F. Sun, and K. Ma. 2009. Species-area

476 relationships explained by the joint effects of dispersal limitation and habitat heterogeneity.

477 Ecology 90:3033-3041.

478 Sindorf, V. 2009. Animal movements: The utilization of white-tailed deer (Odocoileus

479 virginianus) trails by vertebrates as a means of traversing gaps in landscape matrices (PhD). (A.

480 R. K. Petra Vande Zande, ed.). Cornerstone University.

481 Spasojevic, M. J., E. I. Damschen, and S. Harrison. 2014. Patterns of seed dispersal syndromes

482 on serpentine soils: examining the roles of habitat patchiness, soil infertility and correlated

483 functional traits. Plant Ecology \& Diversity 7:401-410. 
484 Thomson, F. J., A. T. Moles, T. D. Auld, and R. T. Kingsford. 2011. Seed dispersal distance is

485 more strongly correlated with plant height than with seed mass. Journal of Ecology 99:1299_

4861307.

487 Wahlberg, N., A. Moilanen, and I. Hanski. 1996. Predicting the occurrence of endangered

488 species in fragmented landscapes. Science 273:1536-1538.

489 White, C., K. A. Selkoe, J. Watson, D. A. Siegel, D. C. Zacherl, and R. J. Toonen. 2010. Ocean

490 currents help explain population genetic structure. Proceedings of the Royal Society of London

491 B: Biological Sciences 277:1685-1694. 


\section{$507 \quad$ Figures and Tables}

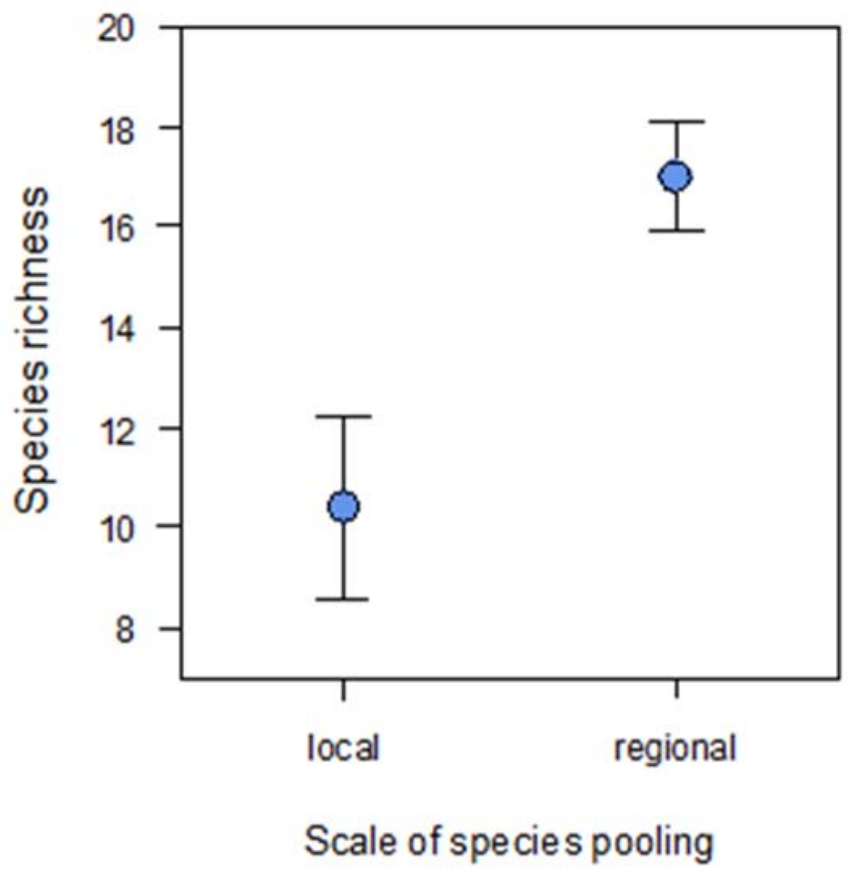

509 Figure 1: Experimental evidence of dispersal limitation via pooling seed banks within (local) or

510 among (regional) habitat patches to enhance dispersal; on average, we see an increase of 7

511 species per habitat patch with regional pooling $\left(F_{1,4}=15.2, P=0.0175\right)$. The data presented here

512 is subsetted from a larger dataset (Germain et al. 2017) to include only sites within the same

513 region as our current survey, and only treatments that received locally-mixed species pools $(5 \mathrm{~m}$

514 spatial scale) and those mixed among sites within the regional extent of our survey (100 m

515 spatial scale). 


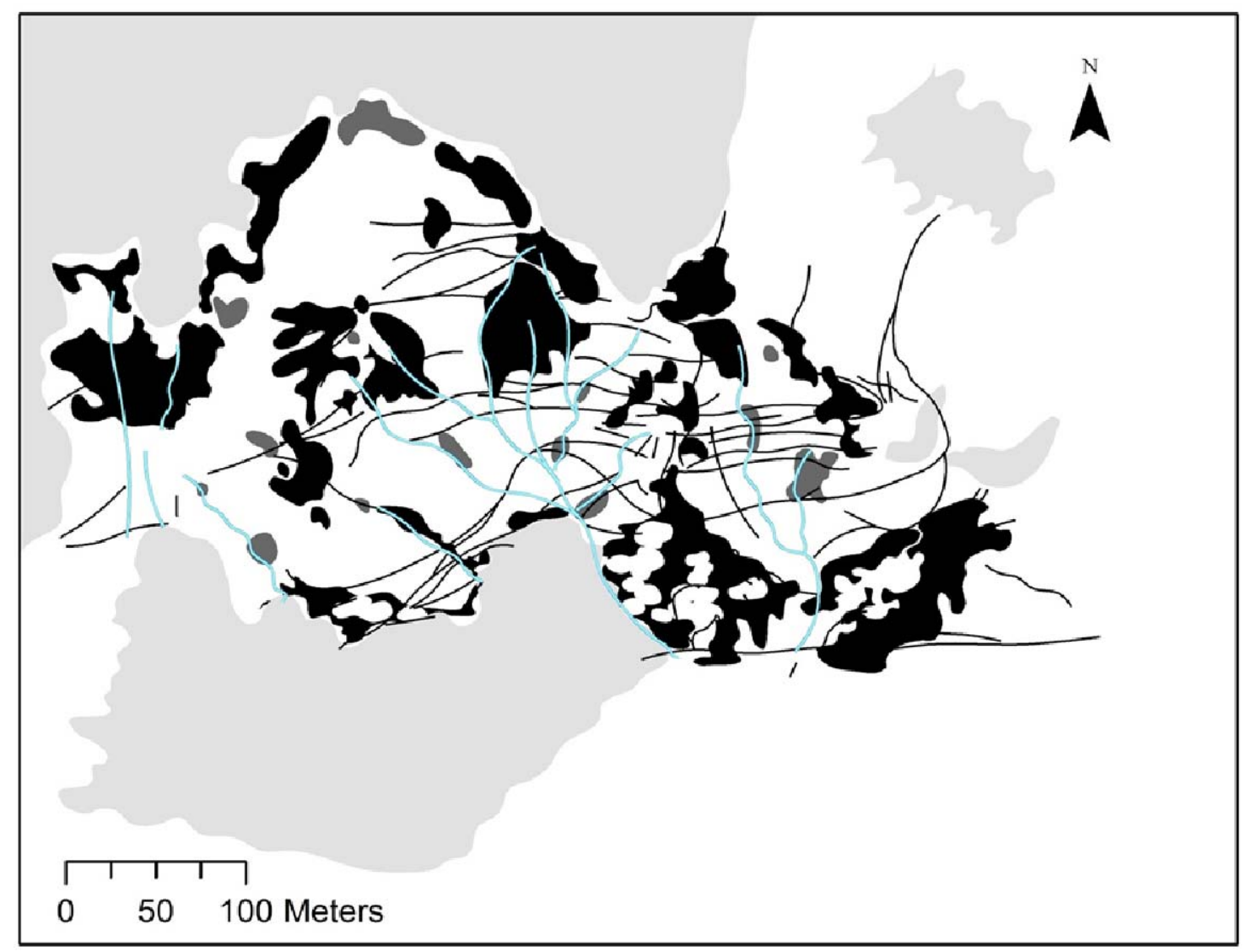

517 Figure 2: Map of sampled (black) and unsampled (dark gray) habitat patches at our 18-ha study

518 site within McLaughlin Reserve, CA. Thin black lines are animal paths and thick blue lines are

519 surface runoff, both traced from satellite imagery. The habitat matrix (white) was non-serpentine

520 soils dominated by European grasses, such as Avena barbata, and the site boundaries were either

521 road or chaparral (light grey). 
(A) Gravity-dispersed

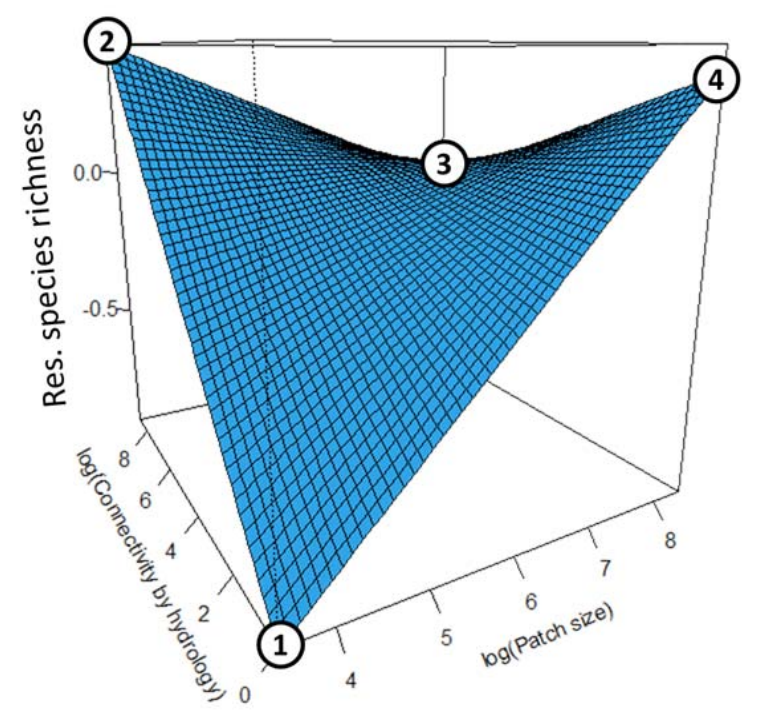

(B) Animal-dispersed

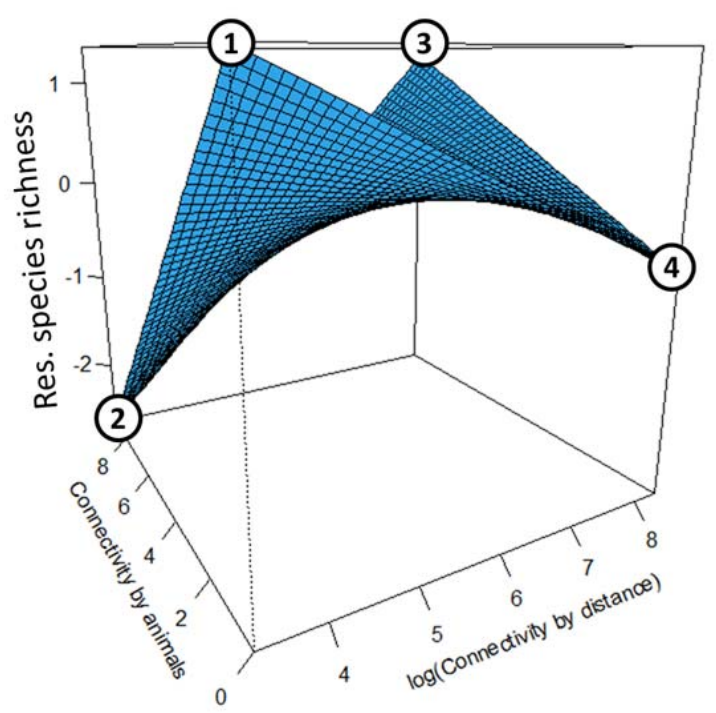

523 Figure 3: Species richness of $(A)$ gravity-dispersed species, as a function of patch size and

524 hydrological connectivity, and of $(B)$ animal-dispersed species, as a function of connectivity by

525 distance and animal connectivity. Numbered points connect different slopes to aid our

526 description of the response surface in main text. Fitted response surfaces of species richness are

527 shown for simplicity, after partialling out fixed effect of patch productivity and random effect of

528 site ID, but residuals are shown in fig. A3. 
534 Table 1: Occupancy of Plantago erecta, a species with a mixed dispersal strategy, is explained

535 by connectivity measures intermediate to those exhibited by species with animal and gravity

536 dispersal

\begin{tabular}{lccc}
\hline Variables & Slope & $\boldsymbol{X}^{\mathbf{2}}$ value & $\boldsymbol{P}$-value \\
\hline size & -2.15 & 0.06 & 0.812 \\
distance & 13.30 & 0.31 & 0.578 \\
animals & 30.99 & 10.44 & $\mathbf{0 . 0 0 1}$ \\
hydrology & 13.64 & 36.29 & $<\mathbf{0 . 0 0 1}$ \\
distance $\times$ animals & -4.70 & 2.15 & 0.143 \\
distance $\times$ hydrology & & & 0.176 \\
size $\times$ hydrology & -2.53 & 1.83 & 0.093 \\
animals $\times$ hydrology & & & \\
distance $\times$ animal $\times$ hydrology & 0.85 & 5.70 & $\mathbf{0 . 0 1 7}$ \\
\end{tabular}

537 Note: Significant $P$-values are in bold typeface. This reduced model provided a significantly

538 better fit to the occupancy data than an intercept-only model, despite requiring an additional 9

539 degrees of freedom (model comparison: $P=0.012$ ). 